\title{
Geração de resíduos na aquisição de vegetais in natura e minimamente processados por serviço de nutrição e dietética de um hospital público
}

\author{
Waste generation in the acquisition of vegetables in nature \\ and minimally processed by nutrition and dietary service of a \\ public hospital
}

\author{
Vanessa Thais Peres Melo ${ }^{1}$, Virgílio José Strasburg ${ }^{2^{*}}$ (D) \\ ${ }^{1}$ Universidade Federal do Rio Grande do Sul (UFRGS), Faculdade de Medicina, Departamento de Nutrição, \\ Porto Alegre/RS - Brasil \\ ${ }^{2}$ Universidade Federal do Rio Grande do Sul (UFRGS), Centro de Estudos em Alimentação e Nutrição - CESAN, \\ Departamento de Nutrição, Porto Alegre/RS - Brasil
}

*Corresponding Author: Virgílio José Strasburg, Universidade Federal do Rio Grande do Sul (UFRGS),

Departamento de Nutrição, Rua Ramiro Barcelos, 2400, $4^{\circ}$ andar, sala 405, CEP: 90035-003, Porto Alegre/RS -

Brasil, e-mail: virgilio_nut@ufrgs.br

Cite as: Melo, V. T. P., \& Strasburg, V. J. (2020). Waste generation in the acquisition of vegetables in nature and minimally processed by nutrition and dietary service of a public hospital. Brazilian Journal of Food Technology, 23, e2019069. https://doi.org/10.1590/1981-6723.06919

\begin{abstract}
Resumo
As atividades na produção de refeições são causadoras de impactos ambientais. Este estudo teve como objetivo avaliar a geração de resíduos provenientes da aquisição de vegetais in natura e vegetais minimamente processados (VMP) para o fornecimento de refeições, em um Serviço de Nutrição e Dietética de um hospital público federal. Trata-se de um estudo do tipo transversal descritivo, retrospectivo, de caráter quantitativo e com utilização de dados secundários, considerando o ano de 2016 e o primeiro semestre de 2017. Como resultado, a média mensal, estimada em $5.545,8 \mathrm{~kg}$ de resíduos das partes não comestíveis de frutas, corresponde a $34,2 \%$ do total adquirido no ano de 2016. Os dados do primeiro semestre de 2017 se mostraram 3,7\% superiores à média de 2016. $\mathrm{Na}$ avaliação das hortaliças, a aquisição de VMP, no período de estudo, projetou uma não geração de 73,8 toneladas de resíduos no serviço. Se esses vegetais fossem comprados in natura, seriam necessários $25,6 \%$ a mais de matérias-primas no ano de 2016 e, também, no primeiro semestre de 2017. Os resultados deste estudo mostram que a aquisição de VMP torna-se uma alternativa para os serviços de alimentação e nutrição.
\end{abstract}

Palavras-chave: Frutas; Hortaliças; Impacto ambiental; Meio ambiente; Resíduos sólidos; Serviços de alimentação.

\begin{abstract}
Meal production activities are responsible for the environmental impact. This study evaluated waste generation from the acquisition of vegetables in nature and minimally processed vegetables (MPV) for meal supply in a Nutrition and Dietetics Service of a federal public hospital. It is a cross-sectional descriptive, retrospective study, of
\end{abstract}


quantitative character, with secondary data considering 2016 and the first semester of 2017. As a result, the estimated monthly mean of waste generation from non-edible parts of fruit was $5545.8 \mathrm{~kg}$, corresponding to $34.2 \%$ of the total acquisition of 2016. Data from the first semester of 2017 showed an increase of $3.7 \%$ higher than the 2016 average. In the evaluation of vegetables, MPV acquisition by the study period projected a non-waste generation of 73,8 tons. If these vegetables were bought in nature would be necessary more $25.6 \%$ of raw material in 2016, and in the first semester of 2017. The results of the present study showed that MPV acquisition becomes an alternative for food and nutrition services.

Keywords: Fruits; Vegetables; Environmental impact; Environment; Solid waste; Food services.

\section{Introdução}

Os hábitos alimentares, como o seu padrão e o tempo dedicado às refeições, vêm sofrendo uma crescente mudança, especialmente ao longo das últimas décadas. Pode ser observada uma grande relação entre o nível econômico e o consumo de alimentos fora dos domicílios, demonstrando que a renda apresenta um papel importante na determinação desta escolha (Claro et al., 2014).

Com vistas a essa realidade, que demanda conveniência e praticidade para compra e consumo de alimentos, a população busca por produtos prontos para o consumo ou que exijam pouco ou nenhum preparo, para serem consumidos com segurança (Moretti, 2007).

Os vegetais minimamente processados (VMP) são alimentos que passam por procedimentos, como limpeza, lavagem, descascamento, corte, embalagem e armazenamento, e apresentam qualidade semelhante à do produto fresco. Devido à sua praticidade, possuem ótimo potencial de comércio, por serem alimentos naturais, de alta qualidade e adequadas condições higiênicas, para o consumo seguro da população (Chitarra, 2000). Atualmente, pesquisadores e a indústria vêm trabalhando em novas tecnologias, com a finalidade de garantir a qualidade sanitária e sensorial dos alimentos, e também para diminuir impactos ao meio ambiente (Alzamora et al., 2016).

O fornecimento de refeições para coletividades pode ocorrer em espaços denominados Unidade de Alimentação e Nutrição (UAN). A função das UANs é promover alimentação e nutrição visando ao fornecimento de refeições balanceadas, seguindo padrões dietéticos e higiênicos, com o intuito de atender às necessidades nutricionais dos comensais.

Vegetais, como frutas, hortaliças e verduras, são utilizados na alimentação humana e, em uma UAN, fazem parte da elaboração dos cardápios em preparações, como saladas e guarnições. Devido aos diversos procedimentos envolvidos na produção e distribuição de refeições, as UANs são causadoras de impactos ambientais, destacando a utilização de grande quantidade de água e energia, e também a geração de volume de resíduos sólidos, especialmente por conta das diversas etapas envolvidas no uso in natura de frutas, verduras e legumes (Abreu \& Spinelli, 2016; Almeida et al., 2015).

Por conta disso, é necessário que as UANs adotem práticas que preservem os recursos naturais e diminuam os danos ao ambiente. Uma dessas possibilidades é a utilização de VMP. Atualmente, o meio ambiente é uma preocupação central, atraindo o interesse mundial independentemente do regime político ou do sistema econômico de cada país (Barthichoto et al., 2013a).

Marques \& Almeida (2013) definem que a produção, o comércio e o consumo de produtos alimentícios são contribuintes cruciais para inúmeros problemas ambientais. De Laurentiis et al. (2017) listaram o desperdício de alimentos como um dos principais itens que influenciam na emissão de gases de efeito estufa (GEE). Esse tipo de resíduo, se não for destinado corretamente para aterros sanitários, passará a se decompor 
e gerar emissões de metano, um GEE de efeitos potentes, que contribui para alterações climáticas de forma significativa (Pirani \& Arafat, 2014).

Considerando os impactos ambientais, relacionados à produção de refeições, este estudo tem por objetivo avaliar e comparar a geração de resíduos provenientes da utilização de vegetais in natura e VMP, no fornecimento de refeições em um Serviço de Nutrição e Dietética (SND) de um hospital público federal, da cidade de Porto Alegre-RS.

\section{Metodologia}

O estudo é do tipo transversal descritivo, retrospectivo, de caráter quantitativo, com utilização de dados secundários (Prodanov \& Freitas, 2013), realizado em um hospital público federal, no município de Porto Alegre-RS. Os dados de aquisição de gêneros foram obtidos dos relatórios gerenciais do serviço de nutrição e dietética (SND) do hospital, contemplando o período do ano de 2016 e primeiro semestre de 2017.

Foram avaliadas variáveis quantitativas relativas ao consumo de Vegetais Minimamente Processados (VMP) e in natura. Utilizou-se listagem dos vegetais utilizados nas refeições diárias, incluindo café da manhã, colação, almoço, café da tarde, jantar e ceia.

O cardápio das refeições - almoço e jantar - é composto por cereal (arroz branco ou integral), leguminosa (feijão ou lentilha), guarnição (hortaliças ou gêneros secos, como farináceos e massas), carnes (proteína animal: bovina, ave, suíno ou peixe), saladas (hortaliças) e fruta como sobremesa. As frutas também podem ser oferecidas, de acordo com prescrição dietética, para os pacientes, nas refeições: café da manhã, colação, café da tarde e ceia.

A seleção dos itens investigados neste estudo considerou o critério da curva $\mathrm{ABC}$, seguindo o modelo utilizado por Strasburg \& Jahno (2017a). Foram elencadas todas as hortaliças e frutas adquiridas in natura ou VMP, sendo considerados todos os itens de aquisição, de cada grupo, até atingir a quantidade mínima de $85 \%$ do total dos alimentos, consumidos em quilogramas $(\mathrm{kg})$, para cada período avaliado.

Para a realização dos cálculos de estimativa da geração de resíduo por meio do Índice de Parte Comestível (IPC), outrora denominado de Fator de Correção, foram utilizados os valores de referência do livro "Técnica Dietética" (Ornellas, 2007). Desses cálculos, são obtidos os percentuais de rendimento dos alimentos. O cálculo para a definição do IPC de um alimento se dá mediante a divisão do peso bruto pelo peso líquido, resultante da retirada das partes não comestíveis do alimento (Abreu \& Spinelli, 2016).

Os dados quantitativos coletados foram transcritos para planilha eletrônica do programa Microsoft Office Excel $2010^{\circledR}$. Foram descritas as frequências absolutas e, ainda, realizados cálculos percentuais, de médias e desvio padrão.

A realização deste estudo foi vinculada ao projeto n. ${ }^{\circ}$ 29759/2014, cadastrado na Plataforma Brasil e aprovado pela Comissão de Pesquisa da Faculdade de Medicina da Universidade Federal do Rio Grande do Sul.

\section{Resultados e discussão}

Do total de 46 alimentos, classificados como hortaliças e frutas, foram selecionados 21 itens pelo critério da curva $\mathrm{ABC}$, agrupados, respectivamente, em frutas in natura, hortaliças in natura e hortaliças VMP, conforme apresentado na Tabela 1. 
Geração de resíduos na aquisição de vegetais in natura e minimamente processados por serviço de nutrição e dietética de um hospital público

Melo, V. T. P., \& Strasburg, V. J.

Tabela 1. Relação de frutas e hortaliças, e frequência de aquisição por SND hospitalar. Porto Alegre-RS, 2016-17.

\begin{tabular}{|c|c|c|c|c|c|c|}
\hline \multirow[b]{2}{*}{ Alimento } & \multicolumn{3}{|c|}{2016} & \multicolumn{3}{|c|}{2017} \\
\hline & $\begin{array}{c}\begin{array}{c}\text { Frequência } \\
\text { (meses/ano)* }\end{array} \\
\end{array}$ & Média (kg) & DP & $\begin{array}{c}\begin{array}{c}\text { Frequência } \\
\text { (meses/ano)* }\end{array} \\
\end{array}$ & Média (kg) & DP \\
\hline Abacaxi & 12 & 793,3 & 303,0 & 6 & 902,0 & 170,0 \\
\hline Banana & 12 & 5760,7 & 2172,5 & 6 & 5948,5 & 1123,0 \\
\hline Laranja & 12 & 4027,3 & 1131,5 & 6 & 4428,3 & 483,5 \\
\hline Maçã & 12 & 2710,0 & 1675,0 & 6 & 2436,8 & 1369,0 \\
\hline Mamão & 12 & 1607,6 & 474,0 & 6 & 1526,8 & 393,0 \\
\hline Tangerina & 5 & 2809,0 & 1999,0 & 2 & 2772,5 & 1024.5 \\
\hline Caqui & 1 & 1865,0 & 0,0 & 3 & 1483,3 & 112,5 \\
\hline Abobrinha & 9 & 985,0 & 607,0 & 6 & 1120,7 & 247,0 \\
\hline Cenoura & 8 & 568,8 & 560,0 & 0 & 0,0 & 0,0 \\
\hline Repolho & 12 & 897,3 & 430,0 & 6 & 779,7 & 212,5 \\
\hline Tomate & 12 & 5687,3 & 1680,0 & 6 & 5475,5 & 829,0 \\
\hline Batata & 12 & 2573,8 & 1707,5 & 6 & 2040,5 & 696,0 \\
\hline Beterraba & 12 & 1057,9 & 559,0 & 6 & 1073,8 & 133,5 \\
\hline Cebola & 12 & 3246,1 & 1570,0 & 6 & 3099,8 & 448,5 \\
\hline Cenoura & 12 & 3614,2 & 1979,5 & 6 & 3018,8 & 485,5 \\
\hline Chuchu & 12 & 2545,8 & 3797,5 & 6 & 2546,2 & 259,0 \\
\hline Moranga & 12 & 2126,6 & 1063,0 & 6 & 2068,8 & 280,0 \\
\hline Alface & 8 & 294,9 & 603,5 & 6 & 1102,3 & 306,0 \\
\hline Chicória & 12 & 174,2 & 100,5 & 4 & 193,0 & 88,5 \\
\hline Couve & 8 & 182,6 & 244,0 & 5 & 193,4 & 133,0 \\
\hline Espinafre & 12 & 861,9 & 467,5 & 6 & 709,7 & 210,5 \\
\hline
\end{tabular}

Legenda: DP: desvio padrão. Obs.: $(*)$ refere-se ao número de meses em que o insumo foi adquirido. Fonte: Autores.

Das sete frutas de maior aquisição, apenas duas variedades (tangerina e caqui) não foram adquiridas em todos os meses do ano de 2016 e no primeiro semestre de 2017, por conta de sua sazonalidade e safra. A utilização de produtos de safra e, no caso específico da tangerina, a qual o estado do Rio Grande do Sul produz o suficiente para a demanda interna, auxilia na redução do impacto ambiental. Pode ser destacado que a produção de alimentos da época requer menor gasto energético, tendo em conta a não necessidade de aquecimento em estufas ou de outros sistemas de proteção, ou favorecimento ambiental para o cultivo fora de safra (Agol et al., 2014). Além disso, a aquisição sazonal evita que os alimentos tenham percorrido longas distâncias, dentro do mesmo país, diminuindo os impactos ambientais relacionados ao transporte (Sustainable Development Commission, 2009).

Dos 14 itens classificados como hortaliças, quatro deles foram adquiridos in natura e os outros 10, na modalidade de VMP. Os itens adquiridos in natura são produtos que exigem pouca manipulação, em relação à retirada de partes não comestíveis. Das hortaliças adquiridas como VMP, somente alface e chicória são utilizados exclusivamente como saladas, sendo as demais utilizadas também como preparo de guarnições, sopas, consomês, saladas cozidas ou, ainda, como tempero (cebola).

A aquisição das frutas in natura, nos períodos de investigação, com a estimativa de geração de resíduos de partes não comestíveis, é apresentada na Tabela 2. 
Geração de resíduos na aquisição de vegetais in natura e minimamente processados por serviço de nutrição e dietética de um hospital público

Melo, V. T. P., \& Strasburg, V. J.

Tabela 2. Aquisição de frutas in natura por um SND hospitalar e estimativa de geração de resíduos. Porto Alegre-RS, 2016-17.

\begin{tabular}{|c|c|c|c|c|c|}
\hline \multirow[b]{2}{*}{ Frutas in natura } & \multirow[b]{2}{*}{ IPC } & \multicolumn{2}{|c|}{2016} & \multicolumn{2}{|c|}{2017} \\
\hline & & $\begin{array}{l}\text { Qtde } \\
\text { (kg) }\end{array}$ & $\begin{array}{c}\text { Total RG } \\
\text { (kg) }\end{array}$ & $\begin{array}{l}\text { Qtde } \\
\text { (kg) }\end{array}$ & $\begin{array}{c}\text { Total RG } \\
(\mathrm{kg})\end{array}$ \\
\hline Abacaxi & 1,89 & 9519 & 4473,9 & 5412 & 2543,6 \\
\hline Banana & 1,53 & 69128 & 23503,5 & 35691 & 12134,9 \\
\hline Laranja & 1,76 & 48328 & 20781,0 & 26570 & 11425,1 \\
\hline Maçã & 1,25 & 32520 & 6504,0 & 14621 & 2924,2 \\
\hline Mamão & 1,63 & 19291 & 7523,5 & 9684 & 3776,8 \\
\hline Tangerina & 1,36 & 14045 & 3651,7 & 5545 & 1441,7 \\
\hline Caqui & 1,06 & 1865 & 111,9 & 4450 & 267,0 \\
\hline Total & & 155887 & 66594,6 & 101973 & 34513,3 \\
\hline
\end{tabular}

Legenda: IPC: índice de Parte Comestível; Qtde: quantidade; Total RG: total de resíduos gerados. Fonte: Autores.

Das frutas elencadas, o abacaxi e o mamão devem ser porcionadas para o atendimento dos pacientes. O abacaxi destaca-se na literatura como a fruta que apresenta o maior fator de IPC, visto que, além da casca, o mesmo apresenta a coroa (Ornellas, 2007). Dentre as frutas entregues, de maneira unitária, e utilizadas também como opção de sobremesa no restaurante do hospital, a banana teve a maior aquisição anual e a maior quantidade de geração de resíduos.

A média mensal estimada da geração de resíduos das partes não comestíveis de frutas foi de $5.545,8 \mathrm{~kg}$, correspondendo a $34,2 \%$ do total adquirido no ano de 2016 . No primeiro semestre de 2017 , a estimativa da média mensal foi de $5.752,2 \mathrm{~kg}$, ou seja, um acréscimo de 3,7\% no total de resíduos. Seguindo essa tendência, ao final do ano de 2017, o resíduo total, proveniente do IPC das frutas, poderia alcançar o montante de $69.026,7 \mathrm{~kg}$. As frutas higienizadas foram adquiridas eventualmente pelo SND, no entanto, representaram apenas $1,2 \%$ do total em quilogramas de frutas adquiridas, no ano de 2016 , e $1,5 \%$ no primeiro semestre de 2017. Deve ser realçado que esse desperdício das partes não consumidas implicou, ainda, na perda dos recursos que foram necessários para produzir esses alimentos, entre os quais, água e energia (Pirani \& Arafat, 2014).

Tabela 3. Aquisição de hortaliças in natura por um SND hospitalar e estimativa de geração de resíduos. Porto AlegreRS, 2016-17.

\begin{tabular}{ccccccc}
\hline \multirow{2}{*}{$\begin{array}{c}\text { Hortaliças } \\
\text { in natura }\end{array}$} & \multirow{2}{*}{ IPC } & & \multicolumn{2}{c}{$\mathbf{2 0 1 6}$} & & \multicolumn{2}{c}{$\mathbf{2 0 1 7}$} \\
\cline { 7 - 7 } \cline { 6 - 7 } \cline { 6 - 7 } & & Qtde & Total RG (kg) & & Qtde & Total RG (kg) \\
\hline Abobrinha & 1,35 & 8865 & 2127,6 & & 6724 & 1613,8 \\
\hline Cenoura & 1,17 & 4550 & 682,5 & & 0,0 & 0 \\
\hline Repolho & 1,72 & 10768 & 3768,8 & 4678 & 1637,3 \\
\hline Tomate & 1,25 & 68248 & 17062,0 & & 32853 & 8213,3 \\
\hline TOTAL & & $\mathbf{9 2 4 3 1}$ & $\mathbf{2 3 6 4 0 , 9}$ & $\mathbf{4 4 2 5 5}$ & $\mathbf{1 1 4 6 4 , 3}$ \\
\hline MÉDIA & & & $\mathbf{1 9 7 0 , 1}$ & & $\mathbf{1 9 1 0 , 7}$ \\
\hline
\end{tabular}

Legenda: IPC: índice de Parte Comestível; Qtde: quantidade; Total RG: total de resíduos gerados. Fonte: Autores.

Das hortaliças in natura, apresentadas na Tabela 3, o tomate representou 73,8\% do total adquirido. Esse item é utilizado como ingrediente para molhos das preparações e, ainda, como salada, sendo oferecido tanto para os usuários do restaurante do hospital quanto para os pacientes. A média mensal de resíduos de IPC das hortaliças no ano de 2016 apresentou um valor apenas 3,1\% superior, quando comparado ao primeiro semestre de 2017. 
Tabela 4. Aquisição de hortaliças higienizadas por um SND hospitalar e estimativa da não geração de resíduos. Porto Alegre, RS, 2016-17.

\begin{tabular}{|c|c|c|c|c|c|}
\hline \multirow{2}{*}{$\begin{array}{c}\text { Hortaliças } \\
\text { higienizadas }\end{array}$} & \multirow{2}{*}{ IPC } & \multicolumn{2}{|c|}{2016} & \multicolumn{2}{|c|}{2017} \\
\hline & & Qtde (kg) & RNG (kg) & Qtde (kg) & RNG (kg) \\
\hline Batata & 1,06 & 30885 & 1853,1 & 12243 & 734,6 \\
\hline Beterraba & 1,75 & 12695 & 4951,1 & 6443 & 2512,8 \\
\hline Cebola & 1,74 & 38953 & 15191,7 & 18599 & 7253,6 \\
\hline Cenoura & 1,17 & 37708 & 5656,2 & 18113 & 2717,0 \\
\hline Chuchu & 1,47 & 30549 & 9775,7 & 15277 & 4888,6 \\
\hline Moranga & 1,4 & 25519 & 5869,4 & 12413 & 2855,0 \\
\hline Alface & 1,21 & 2359 & 401,0 & 6614 & 1124,4 \\
\hline Chicória & 1,4 & 2090 & 543,4 & 772 & 200,7 \\
\hline Couve & 1,91 & 1461 & 482,1 & 967 & 319,1 \\
\hline Espinafre & 1,78 & 10343 & 4550,9 & 4258 & 1873,5 \\
\hline TOTAL & & 192562 & 49274,6 & 95699 & 24479,3 \\
\hline MÉDIA & & 16046,8 & 4106,2 & 15949,8 & 4079,9 \\
\hline
\end{tabular}

Legenda: kg: quilogramas; IPC: índice de Parte Comestível; Qtde: quantidade; Total RNG: total de resíduos não gerados. Fonte: Autores.

De acordo com a Tabela 4, se os insumos que foram adquiridos como VMP fossem comprados in natura, seriam necessários $25,6 \%$ a mais de matérias-primas tanto no ano de 2016 como no primeiro semestre de 2017.

Os vegetais in natura geram uma grande quantidade de resíduos por partes não aproveitáveis. Com a aquisição das hortaliças folhosas, na modalidade VMP, a estimativa de não geração de resíduos, nesse período de 18 meses, é de 9.495,2 kg. O estudo de Redin \& Strasburg (2016) apontou que, no caso dos folhosos, o maior período de armazenamento relacionou, também, um maior valor no IPC destes produtos. A utilização dos insumos in natura também foi responsável pela maior quantidade de resíduos, em estudo que avaliou o cálculo de ecoeficiência nos insumos, utilizados em restaurantes universitários (Strasburg \& Jahno, 2017a)

Para essa quantidade extra de insumos, haveria a necessidade, também, de se utilizar mais espaço físico para armazenamento, incluindo câmaras refrigeradas. Além disso, para o processamento dos alimentos, ocorreria uma maior necessidade de pessoas e maior uso de água e saneantes, os quais irão gerar efluentes (Redin \& Strasburg, 2016).

Embora o preço dos VMP seja superior, o custo final torna-se semelhante ao da aquisição dos alimentos in natura, em consequência do menor investimento com área física e equipamentos, mão de obra e energia (Proença, 2009). A aquisição desses alimentos atenua a carga de trabalho dos funcionários do setor de pré-preparo, visto que as etapas iniciais são eliminadas, diminuindo a área necessária para a disposição temporária de alimentos. Outra vantagem é a diminuição de possíveis problemas no recebimento dos gêneros alimentícios de qualidade inferior à solicitada (Degiovanni et al., 2010).

Em vários países da Europa Ocidental e nos Estados Unidos da América, processos produtivos com inovação tecnológica, como o uso de vegetais pré-elaborados, são mais comumente utilizados, por empresas de alimentação para coletividades (Proença, 1999; Rodgers, 2007). No Brasil, no entanto, tem-se maior aquisição dos vegetais na forma in natura (Kawasaki et al., 2007). Com a maior utilização dos VMP, seriam reduzidos os resíduos gerados nas UANs.

O estudo de Colares \& Figueiredo (2012) identificou que os resíduos de alimentos, provenientes de sobras, restos e rejeitos de pré-preparo, corresponderam a $88 \%$ do total da composição gravimétrica em uma UAN, na cidade do Rio de Janeiro-RJ. Em uma UAN de Goiânia, foi identificado que, do total da geração dos resíduos orgânicos, a etapa de preparo foi responsável por $55,2 \%$ e a distribuição das refeições, por mais 39\% (Araújo \& Carvalho, 2015). Van Waning (2010) descreveu, em seu estudo, que os restos de alimentos representaram $60,3 \%$ do total de geração de resíduos de serviços de hotelaria e restauração. 
Spinelli \& Cale (2009) descreveram que $87,8 \%$ do total de resíduos produzidos em uma UAN tinha como destino aterros sanitários e lixões, e os outros $12,2 \%$ eram descartados em coleta seletiva para reciclagem. Porém, com um programa de avaliação e destino dos resíduos, $74,5 \%$ dos resíduos, que eram encaminhados para os aterros e lixões, poderiam ser utilizados para a compostagem, e apenas os 13,36\% restantes poderiam ser encaminhados para os aterros sanitários. Os resíduos, caracterizados como orgânicos, são grandes geradores de chorume. O chorume é um líquido escuro, que contém alta carga orgânica em decomposição e provoca um impacto ambiental acentuado, principalmente em relação à poluição das águas, podendo prejudicar a fauna e a flora aquática (Sousa et al., 2015).

Barthichoto et al. (2013b), descreveram em sua pesquisa, que, dos 32 restaurantes pesquisados, 25\% realizavam coleta seletiva. Destes, $87,5 \%$ faziam separação de latas, plásticos, papel, metal e resíduos orgânicos, e 12,5\% fazia a separação dos resíduos sólidos recicláveis dos resíduos orgânicos.

Um estudo de Pospischek et al. (2014), que avaliou as ações de sustentabilidade ambiental em restaurantes comerciais, mostrou que, em 56,2\% dos casos, o destino dos resíduos orgânicos era para cooperativas e 43,8\% para a prefeitura. Araújo \& Carvalho (2015) identificaram, num estudo numa UAN da cidade de Goiânia, que $73,2 \%$ dos resíduos orgânicos gerados foram destinados para ração animal. Esses registros corroboram com Strasburg \& Jahno (2017b), que destacaram, no contexto de produções científicas brasileiras, que as abordagens, relacionadas com questões de desperdício de alimentos, estão envolvidas especialmente com as etapas de preparação e de consumo de refeições. Colares et al. (2018) destacam, em seu estudo sobre boas práticas ambientais para serviços de alimentação, um bloco específico para avaliar 33 itens relacionados com a gestão de resíduos sólidos.

Segundo o artigo de Peregrin (2011), as decisões realizadas por gerentes de operações de serviços de alimentação podem ter impactos significativos na economia e no meio ambiente. Dessa forma, especialmente para UANs de grande porte localizadas em centros urbanos, o uso de VMP passa a ser uma alternativa viável, na gestão do serviço, por otimizar as etapas de trabalho nas cozinhas e, também, por diminuir a quantidade de resíduos relacionados com as etapas de preparação de refeições.

A American Dietetic Association incentiva as práticas ambientais que visam a preservação dos recursos naturais, minimização da quantidade de resíduos e sustentabilidade no sistema alimentar. A conservação e a proteção dos recursos naturais contribuem para a sustentabilidade no presente e no futuro (Harmon \& Gerald, 2007).

\section{Conclusões}

O presente estudo comparou a estimativa da geração de resíduos, nas modalidades de aquisição de vegetais in natura e minimamente processados, para o fornecimento de refeições de um Serviço de Nutrição e Dietética (SND) hospitalar.

Os resultados deste estudo mostraram que a maior quantidade de aquisição de hortaliças minimamente processadas proporcionou uma menor geração de resíduos orgânicos na produção de refeições, o que implica numa melhor logística de coleta de resíduos nesse hospital. Além disso, a aquisição dos VMP contribui para a redução do uso de água e produtos saneantes, provocando, assim, menos impactos ambientais por parte do SND. Como limitação deste estudo, deve ser considerado o fato de se utilizarem referências da literatura para a realização dos cálculos. Nesse sentido, muitos fatores estão envolvidos nas etapas de preparo dos alimentos, como a qualidade dos insumos, os utensílios utilizados e a habilidade dos manipuladores.

A utilização de vegetais minimamente processados por serviços de alimentação para coletividade, especialmente nos locais com grande demanda de insumos, passa a ser uma alternativa interessante para a redução de resíduos e impactos ambientais, localizados em grandes centros urbanos. Sugere-se que mais estudos, dessa natureza, possam ser realizados por outros serviços de alimentação, a fim de aprimorar a abordagem desse tema de investigação. 


\section{Referências}

Abreu, E. S., \& Spinelli, M. G. N. (2016). A unidade de alimentação e nutrição. In E. S. Abreu, M. G. N. Spinelli, \& A. M. S. Pinto. Gestão de unidades de alimentação e nutrição: Um modo de fazer (5. ed., 378 p.). São Paulo: Metha.

Agol, D., Latawiec, A. E., \& Strassburg, B. B. N. (2014). Evaluating impacts of development and conservation projects using sustainability indicators: opportunities and challenges. Environmental Impact Assessment Review, 48, 1-9. http://dx.doi.org/10.1016/j.eiar.2014.04.001

Almeida, J. L., Santana, K. B., \& Menezes, M. B. C. (2015). Sustentabilidade em unidades de alimentação e nutrição. Revista Encontro Internacional de Formação de Professores e Fórum Permanente de Inovação Educacional, 8(1), 1-15.

Alzamora, S. M., López-Malo, A., Tapia, M. S., \& Welti-Chanes, J. (2016). Minimally processed foods. In B. Caballero, P.M. Finglas, \& F. Toldrá. Encyclopedia of food and health (pp. 767-771). Amsterdam: Elsevier.

Araújo, E. M. L., \& Carvalho, A. C. M. S. (2015). Sustainability and waste generation in a food and nutrition unit in Goiania-GO, Brazil. Demetra, 10(4), 775-796. http://dx.doi.org/10.12957/demetra.2015.17035

Barthichoto, M., Matias, A. C. G., \& Spinelli, M. G. N. (2013a). Avaliação de práticas sustentáveis na produção de refeições segundo o tipo de gestão. Nutrição em Pauta, 21(122), 25-29.

Barthichoto, M., Matias, A. C. G., Spinelli, M. G. N., \& Abreu, E. S. (2013b). Responsabilidade ambiental: perfil das práticas de sustentabilidade desenvolvidas em unidades produtoras de refeições do bairro de Higienópolis, município de São Paulo. Qualit@s, 14(1), 1-12.

Chitarra, M. I. F. (2000). Processamento mínimo de frutos e hortaliças (113 p.). Lavras: UFLA/FAEPE.

Claro, R. M., Baraldi, L. G., Martins, A. P., Bandoni, D. H., \& Levy, R. B. (2014). Evolução das despesas com alimentação fora do domicílio e influência da renda no Brasil, 2002/2003 a 2008/2009. Cadernos de Saude Publica, 30(7), 1-9. PMid:25166939. Colares, L. G. T., \& Figueiredo, V. O. (2012). Gestão de resíduos sólidos gerados na produção de refeições. Nutrição em Pauta, 114, 19-24.

Colares, L. G. T., Figueiredo, V. O., \& Ferreira, A. A., \& Oliveira, A. G. M. (2018). Lista de verificação de boas práticas ambientais para os serviços de alimentação: elaboração, validação de conteúdo e confiabilidade interavaliadores. Brazilian Journal of Food Technology, 21(e2017066)

De Laurentiis, V., Hunt, D. V. L., Rogers, C. D. F. (2017). The little book of low carbon eating in the city. Lancaster: Lancaster University. Recuperado em 2019, Março 3, de http://www.energy.soton.ac.uk/files/2017/12/littlebookoflowcarboneatinginthecity.pdf

Degiovanni, G. C., Japur, C. C., Sanches, A. P. L. M., Mattos, C. H. P. S., Martins, L. S., Reis, C. V., \& Vieira, M. N. C. M. (2010). Hortaliças in natura ou minimamente processadas em unidades de alimentação e nutrição: quais aspectos devem ser considerados na sua aquisição? Revista de Nutrição, 5(23), 813-822. http://dx.doi.org/10.1590/S1415-52732010000500011

Harmon, A. H., \& Gerald, B. L., \& American Dietetic Association (2007). Position of the American Dietetic Association: Food and nutrition professionals can implement practices to conserve natural resources and support ecological sustainability. Journal of the American Dietetic Association, 107(6), 1033-1043. PMid:17571455. http://dx.doi.org/10.1016/j.jada.2007.04.018

Kawasaki, V. M., Cyrillo, D. C., \& Machado, F. M. S. (2007). Custo-efetividade da produção de refeições coletivas sob o aspecto higiênico-sanitário em sistemas cook-chill e tradicional. Revista de Nutrição, 2(20), 129-138. http://dx.doi.org/10.1590/S1415-52732007000200002

Marques, C. P., \& Almeida, D. (2013). A path model of attitudinal antecedents of green purchase behavior. Economia e Sociologia, 6(2), 135.

Moretti, C. L. (2007). Manual de processamento mínimo de frutas e hortaliças (531 p.). Brasília: Embrapa Hortaliças.

Ornellas, L. H. (2007). Técnica dietética: seleção e preparo de alimentos (7. ed., 276 p.). São Paulo: Atheneu.

Peregrin, T. (2011). Sustainability in foodservice operations: An update. Journal of the American Dietetic Association, 111(9), 1286-1294, 1288, 1290 passim. PMid:21872690. http://dx.doi.org/10.1016/j.jada.2011.07.017

Pirani, S. I., \& Arafat, H. A. (2014). Solid waste management in the hospitality industry: a review. Journal of Environmental Management, 146, 320-336. PMid:25194519. http://dx.doi.org/10.1016/j.jenvman.2014.07.038

Pospischek, V. S., Spinelli, M. G. N., \& Matias, A. C. G. (2014). Avaliação de ações de sustentabilidade ambiental em restaurantes comerciais localizados no município de São Paulo. Demetra, 9(2), 595-611.

http://dx.doi.org/10.12957/demetra.2014.8822

Prodanov, C. C., \& Freitas, E. C. (2013). Metodologia do trabalho científico: Métodos e técnicas da pesquisa e do trabalho acadêmico. Novo Hamburgo: Feevale. Recuperado em 17 de março de 2017, de www.feevale.br/institucional/editorafeevale/metodologia-do-trabalho-cientifico---2-edicao

Proença, R. P. C. (1999). Novas tecnologias para a produção de refeições coletivas: recomendações de introdução para a realidade brasileira. Revista de Nutrição, 12(1), 43-53. http://dx.doi.org/10.1590/\$1415-52731999000100004

Proença, R. P. C. (2009). Inovação tecnológica na produção de alimentação coletiva (3a ed., 136 p.). Florianópolis: Insular.

Redin, C., \& Strasburg, V. J. (2016). Fatores relacionados à qualidade de folhosos utilizados nas refeições de um Restaurante Universitário do Sul do Brasil. Disciplinarum Scientia, 17(3), 361-372.

Rodgers, S. (2007). Innovation in food service technology and its strategic role. International Journal of Hospitality Management, Houston, 26(4), 899-912. http://dx.doi.org/10.1016/j.ijhm.2006.10.001 
Geração de resíduos na aquisição de vegetais in natura e minimamente processados por serviço de nutrição e dietética de um hospital público

Melo, V. T. P., \& Strasburg, V. J.

Sousa, M. C., Dos Anjos, D. A., Sales, E. M., \& Andrade, M. R. A. (2015). Processos de tratamento do chorume e reaproveitamento: Uma revisão. In Anais do $5^{\circ}$ Encontro Regional de Química, Anais do $4^{\circ}$ Encontro Nacional de Química (Vol., 3, No. 1, pp. 1147). São Paulo: Blucher. http://dx.doi.org/10.5151/chenpro-5erq-am6

Spinelli, M. G. N., \& Cale, L. R. (2009). Avaliação de resíduos sólidos em uma unidade de alimentação e nutrição. Revista Simbio-Logias, 2(1), 21-30.

Strasburg, V. J., \& Jahno, V. D. (2017a). Application of eco-efficiency in the assessment of raw materials consumed by university restaurants in Brazil: A case study. Journal of Cleaner Production, 161, 178-187. http://dx.doi.org/10.1016/j.jclepro.2017.05.089

Strasburg, V. J., \& Jahno, V. D. (2017b). Paradigmas das práticas de gestão ambiental no segmento de produção de refeições no Brasil. Engenharia Sanitaria e Ambiental, 22(1), 3-12. http://dx.doi.org/10.1590/s1413-41522017155538

Sustainable Development Commission. (2009). Setting the table: advice to government on priority elements of sustainable diets (57 p.). Recuperado em 2019, Março 3, de http://hdl.handle.net/10023/2213

Van Waning, A. (2010). Waste characterization study. Chicago: Department of Environment. Recuperado em 2017, Dezembro 20, de www.cityofchicago.org/dam/city/depts/doe/general/RecyclingAndWasteMgmt_PDFs/

WasteAndDiversionStudy/WasteCharacterizationReport.pdf

Financiamento: Nenhuma.

Received: Mar. 09, 2019; Accepted: Oct. 07, 2019 